\title{
El trabajo infantil rural en México y Argentina. El caso de dos complejos agroindustriales $^{1}$
}

\author{
The Rural Child Labor in México and \\ Argentina. Two Agro-industrial Scenarios
}

\section{O trabalho infantil rural no México e na Argentina. O caso de dois complexos agroindustriais}

\section{Sarai Miranda Juárez ${ }^{2}$}

Investigadora invitada modalidad posdoctoral en el Instituto Gino Germani, Universidad de Buenos Aires, Argentina

saraimirandaj@gmail.com

\section{Daniel Alberto $\mathbf{R e}^{3}$}

Becario posdoctoral CONICET Universidad Nacional de Misiones en conjunto con el Instituto Gino Germani, Universidad de Buenos Aires, Argentina

danielalbertore@gmail.com

Recibido: $27 / 08 / 14$

Aprobado: 14/04/15

1 El presente artículo es resultado de las investigaciones doctorales de los autores, llevadas a cabo de 2008 a 2012 en el caso de la floricultura mexicana, realizada por Sarai Miranda; y de 2007 a 2013 en el caso del tabaco argentino realizada por Daniel Re.

2 Licenciada en Economía, Maestra en Ciencias Sociales con especialidad en Desarrollo Municipal y Doctora en Estudios de Población.

3 Licenciado en Sociología, Maestro en Ciencias Sociales del Trabajo y Doctor en Ciencias Sociales. 


\title{
Resumen
}

El artículo describe las características y el tipo de trabajo que realizan niños y niñas en las actividades de la floricultura y la tabacalera. Busca dar cuenta de dos realidades socioeconómicas atravesadas por un mismo proceso global: el uso de la mano de obra asalariada dentro de los "nuevos" esquemas de producción que imponen los complejos agroindustriales (CAI). En la agricultura latinoamericana son las actividades estacionales altamente demandantes de mano de obra asalariada donde la presencia de mujeres y niños adquiere protagonismo. En muchos casos son usados como complemento de la mano de obra familiar, pero en otros, niños y niñas buscan el empleo para incrementar los ingresos económicos del hogar. La metodología utilizada es de corte cualitativo.

Palabras clave: complejos agroindustriales; trabajo infantil; Argentina; México.

\begin{abstract}
This article describes the characteristics and type of work done by children in activities such as floriculture and tobacco industry. It aims to give an account of two socioeconomic realities that are traversed by the same global process: the "new" model of production imposed by the agro-industrial complexes. In Latin American agriculture, there are seasonal activities that depend on wage labor and temporary workers in which women and children presence plays a main role. In many cases, children help their parents and contribute to the family labor; but in other cases, both boys and girls are workers themselves attempting to increase the household incomes. The methodology used is qualitative.
\end{abstract}

Keywords: agro-industrial complex; child labor; Argentina; Mexico.

\section{Resumo}

O artigo descreve as caraterísticas e o tipo de trabalho realizado por crianças em atividades da floricultura e do tabaco. Tenta abordar duas realidades socioeconômicas atravessadas pelo mesmo processo global: o uso de mão-de-obra assalariada nos "novos" esquemas de produção impostos pelos complexos agroindustriais (CAI). Nas atividades estacionais da agricultura latino-americana, altamente demandantes de mão-de-obra assalariada, a presença de mulheres e crianças adquire protagonismo. Em muitos casos esta mão-deobra é usada como complemento da força de trabalho familiar, porém também meninos e meninas procuram emprego para incrementar a renda do lar. A metodologia usada é de corte qualitativa.

Palavras-chave: complexos agroindustriais; trabalho infantil, Argentina, México.

Este trabajo está bajo la licencia Creative Commons Attribution 3.0 


\section{Introducción}

La Organización Internacional del Trabajo estimó que América Latina y el Caribe fue la tercera región del mundo con mayor porcentaje de niños y niñas trabajadores, registrando casi 12,5 millones de niñas y niños en esta situación. La mayoría de este grupo poblacional trabajó en la agricultura (48\%), con importantes diferencias: Región Andina, 61,5\%; Mesoamérica, 42,7\%; Cono Sur, 38,4\% (OIT 2013). Otros se desempeñaron en sectores como la minería, los basureros, el trabajo doméstico, la cohetería, las elaboraciones de ladrillos y la pesca, consideradas actividades de alto riesgo.

En la agricultura latinoamericana, la presencia de mujeres y niños adquiere protagonismo en las economías de tipo campesino y las actividades altamente demandantes de mano de obra asalariada y estacional. En muchos casos son usados como complemento de la mano de obra familiar (trabajan junto a sus padres); pero en otros, son los niños y niñas quienes buscan el empleo para incrementar los ingresos económicos del hogar.

Durante las últimas décadas, se han realizado investigaciones relativas al trabajo infantil en el agro latinoamericano. Para el caso argentino, por ejemplo, Susana Aparicio sostiene que los niños y niñas que viven en escenarios rurales incorporan desde temprana edad el patrón de conducta asociado a la inclusión en el mundo del trabajo, el cual adquiere características diversas, dependiendo del tipo de actor social al que se haga referencia. Es decir, que la problemática y la concepción que se tenga sobre el trabajo infantil serán diferentes si hablamos de un pequeño productor familiar (campesino) o si nos referimos a un asalariado rural. En este sentido, a la hora de definir la dinámica dentro del grupo familiar, señala que para un campesino (o colono, como lo nombra en el estudio), la "ayuda familiar" es una de las principales estrategias de los hogares, valorada incluso positivamente. En cuanto a los asalariados rurales, la situación se presenta diferente. En la mayoría de los casos, los hijos continúan la misma trayectoria de vida que sus padres, iniciándose tempranamente en el mercado laboral por necesidades económicas de las familias (Aparicio 2007).

Siguiendo esta línea de trabajo, proponemos marcar una diferencia entre trabajo infantil asalariado y trabajo infantil campesino o familiar. El primero es el que se hace presente dentro de los Complejos Agroindustriales (CAI) que aquí abordamos. Si bien, sobre todo en el caso de México, puede encontrarse población de origen campesino e indígena, la tendencia reciente ha sido la incorporación de grupos familiares enteros al trabajo asalariado (Morett y Cosío 2004). Los casos que abordamos corresponden a la actividad tabacalera en la provincia argentina de Jujuy y a la floricultura comercial de exportación en la región de sur del Estado de México. Escenarios ambos donde la participación de la población infantil adquiere características particulares, pero comparten elementos comunes como la asalarización, la estacionalidad y la segmentación por tareas dentro del proceso productivo.

En este sentido, el presente artículo se propone dar cuenta y analizar (en forma comparada) dos realidades socioeconómicas atravesadas por un mismo proceso global: el uso de la mano de obra asalariada dentro de los nuevos esquemas de producción que imponen los complejos agroindustriales en este tipo de 
producciones. Si bien ambos casos forman parte de distintos CAI y están situados en hemisferios diferentes, cada escenario presenta interesantes particularidades y algunas similitudes que permiten compararlos. Por ejemplo, ambos han experimentado importantes procesos de modernización, como la incorporación de nuevas tecnologías (agroquímicos y variedades) con un impacto directo en las características del mercado de trabajo, definiendo una forma estandarizada de producción, con tareas y nuevos puestos de trabajo. Buscamos describir los procesos en cada una de ellas, las características que asume la mano de obra asalariada, las modalidades de contratación existentes en uno y otro escenario, y cómo se da la "ayuda" y participación de niños y niñas.

Para alcanzar el objetivo recurrimos a diversas fuentes de información. En primera instancia, hemos analizado materiales bibliográficos referentes a la actividad tabacalera y la floricultura. Trabajamos con datos estadísticos de organismos oficiales (Instituto Nacional de Estadísticas y Censos en Argentina, y el Instituto Nacional de Estadística, Geografía e Informática en México). El análisis hace especial énfasis en los datos obtenidos directamente en el trabajo de campo. Los insumos que se utilizan provienen de entrevistas en profundidad y entrevistas semiestructuradas a distintos actores sociales involucrados en tales actividades agroindustriales ${ }^{4}$.

Presentado en tres ejes temáticos, el artículo analiza, en primer lugar, los cambios experimentados dentro de cada escenario en particular, y presta especial atención a los "nuevos" escenarios productivos y las modificaciones que impone sobre los procesos de trabajo. En segundo lugar, da cuenta de las características particulares de cada mercado de trabajo, analizando las formas de contratación y la remuneración de los asalariados transitorios, tanto en la floricultura como en la actividad tabacalera. Finalmente, da cuenta de algunas de las características que asume la "ayuda" familiar y el trabajo infantil en cada caso, describiendo tareas, formas de remuneración y momentos del ciclo productivo donde se hace presente el trabajo de niños y niñas.

\section{Cambios recientes en el proceso de producción y en el trabajo agrario}

A finales de la década de 1970 tanto la economía argentina como la mexicana experimentaron considerables cambios en sus modelos económicos, con graves consecuencias para gran parte de su población. La apertura de los mercados y las políticas públicas de desregulación fueron una constante en ambas economías. Los costos "no deseados" de este modelo pueden resumirse básicamente en un aumento de la desocupación, la precarización laboral, el empobrecimiento y la marginalización de amplios sectores de la población. En el ámbito rural, este modelo de acumulación no solo se caracterizó por un aumento en la escala de producción, un mayor uso de insumos y "nuevos" paquetes tecnológicos, sino también por la generalización de los denominados CAI.

4 Los insumos de información cualitativa provienen de las tesis doctorales de los autores. Para el caso de México se realizaron durante el primer cuatrimestre de 2010, en tanto que para el caso de Argentina se levantó en diferentes momentos desde el 2007 al 2012. 
Sin ser un concepto novedoso, empleado desde la década de 1950 para explicar la agroindustria (agribusiness) y dar cuenta de las relaciones insumo-producto, los CAI han vuelto a adquirir protagonismo por sus implicancias mayormente negativas (Rossi y León 2008). Su rol no es solamente proveer insumos y ocuparse del procesamiento industrial de los productos, sino que, además, dicen cómo y cuánto se debe producir, subordinando así a los demás actores sociales (productores y asalariados) bajo distintos grados de verticalidad.

En la floricultura5, por ejemplo, la cadena productiva se estructura "hacia atrás":

\begin{abstract}
Conlleva vínculos con los proveedores de semillas y esquejes, abonos, herbicidas y pesticidas, que generalmente son empresas productoras de agroquímicos. Otros insumos primarios, aunque no de consumo generalizado, son los requeridos para la producción en condiciones de invernadero, que incluyen plásticos, estructuras metálicas y de aluminio así como estacas y grapas, para mantener la flor en posición vertical (Vargas 2007, 281-282).
\end{abstract}

Mientras que "hacia delante" intervienen grandes corporaciones filiales que se hacen cargo del empaque, la transportación, la comercialización nacional e internacional y otras que en algunos casos proveen asistencia técnica (Fenner y Gebauer 1992), así como florerías, empresas de decoración y consumidores directos (Vargas 2007).

En la producción de tabaco jujeña, podemos encontrar dos grandes empresas (Alliance One Tobacco y Massalin Particulares) que como núcleos del complejo determinan los patrones y formas de producción. Estos núcleos o polos integradores responden a empresas internacionales ${ }^{6}$ y tienen la capacidad de determinar qué, cuánto, cuándo y dónde se han de producir y comerciar las materias primas. Es decir, tienen el poder de moldear y pautar los tiempos sobre las unidades de producción agrarias.

Estos modelos de acumulación orientados a la exportación y la implementación de imperativos de eficiencia económica como las flores y el tabaco comparten elementos en común, pues han exigido profundas transformaciones en los modos y formas de producción. Por un lado, en la tabacalera se observa en la modificación de las estufas tradicionales de leña a gas natural y en el reemplazo

5. Las más recientes mediciones indican que el cultivo de flores de corte en la zona sur del Estado de México se extiende en una superficie sembrada promedio de once mil hectáreas; esta superficie representa $0,6 \%$ del total de la superficie agrícola estatal y $86 \%$ de la superficie total dedicada a la floricultura nacional. La región aporta $80 \%$ de las flores que exporta el país, y su comercialización genera una derrama económica anual de 5.995 millones de pesos (617 millones de pesos solo durante la temporada del Día de Muertos), ganancias que representan más de la cuarta parte de la derrama económica que genera el campo mexiquense (30\%) (Orozco 2007).

6 La producción mundial de tabaco presenta una fuerte estructura oligopólica, con más de cien países productores diseminados en diferentes continentes, y solo cuatro empresas internacionales que compran y distribuyen tabaco en todo el mundo: Nacional Tobacco (empresa estatal china) con casi 30\% del mercado, Japan Tobacco (de Japón) con 16\%, Philip Morris (de Estados Unidos) con $17 \%$, y British American Tobacco (de Inglaterra) con 16\% del mercado. El 21\% restante corresponde a otras empresas distribuidas en diferentes partes del mundo (INTA 2008). De estas empresas, tanto Philip Morris como British American Tobacco, a través de Alliance One y otras empresas locales, compran, comercializan y son los agentes fijadores de precio en Brasil, Argentina, Paraguay y Uruguay, por nombrar los más importantes (Re 2013). 
de estas por los hornos para curado a granel (bulk curing)7, con un impacto positivo en los rendimientos y en la calidad final del producto. En la florícola, por su parte, se observa un uso intensivo de recursos tales como la energía, la innovación tecnológica y el capital.

La floricultura comercial del sur del Estado de México representa una actividad económica altamente vinculada a los mercados internacionales, no solo como expendedora de flores sino también como consumidora de insumos de producción provenientes del extranjero. Lo mismo sucede en el caso tabacalero, donde el paquete tecnológico incluye una serie de insumos, como una cuantiosa cantidad de agrotóxicos (sobre todo de importación), semillas (nuevas variedades), estufas a gas, maderas, plásticos, clavos, etc.

Sin embargo, a pesar del cambio tecnológico y la incorporación de nuevas tecnologías (algunas ahorradoras de mano de obra), ambas producciones se caracterizan por presentar una gran demanda de trabajadores dentro del proceso productivo. Según datos publicados por la Dirección de Economía Agraria de Argentina (Corradini 2005), para una hectárea de tabaco en la provincia de Jujuy, se calcula alrededor de 10 jornales permanentes, 4 jornales transitorios para la preparación de los almácigos, 33 jornales transitorios para la preparación del suelo y plantación, y 83 jornales transitorios para la cosecha. Lo que da entre 120 y 130 jornales por hectárea promedio8.

En el caso de la floricultura de exportación en México, los patrones productivos y el mercado de trabajo se comportan de manera similar al CAI tabacalero en Jujuy. Las estimaciones realizadas calculan que para la siembra, corte y poscosecha de flores, solo en el municipio de Villa Guerrero de forma permanente se emplean aproximadamente siete empleados por hectárea cultivada, es decir, 84 mil personas empleadas en actividades relacionadas con la producción floral, cantidad que en temporadas de alta producción llega a triplicarse.

Como vemos, a pesar de haber incorporado alta tecnología -mucha de esta ahorradora de mano de obra- ambas producciones siguen demandando altos niveles de mano de obra asalariada y estacional, concentrada en determinados momentos del ciclo productivo. Es allí donde la presencia de familias enteras dispuestas a emplearse en las temporadas de mayor demanda de trabajo adquiere protagonismo.

\section{Las formas de contratación dentro del complejo agroindustrial y los asalariados transitorios}

La literatura especializada (Bartra 1974; Murmis 1994) ha documentado la forma en que todo modelo de intensificación agroindustrial se acompaña hoy en día de los problemas tradicionales de los mercados de trabajo así como de la emergencia de nuevas dinámicas laborales a su alrededor, en los que la tendencia

7 Una de las principales ventajas de estos es prescindir del encañado tradicional (tarea que se realiza luego de la cosecha y consiste en atar manualmente y de forma individual las hojas de tabaco a una vara para ser llevadas a las estufas), lo que reduce jornales de trabajo y permite el secado de una gran cantidad de hojas en menor tiempo y en forma homogénea.

8 Dependiendo del tamaño y la tecnología utilizada por el productor, en algunos casos se llega a demandar casi 170, ya que cuanto más pequeña y menos eficiente sea en términos productivos, más jornales por hectárea demandará una finca. 
se dirige al deterioro de las condiciones de contratación y uso del factor trabajo a través de la profundización de modalidades como trabajo a destajo y agricultura por contrato.

Una constante en diversos espacios agrícolas latinoamericanos actuales es la mercantilización y flexibilización de la mano de obra, lo que ha llevado a la diversificación del trabajo asalariado y, sobre todo, a la constante presencia de la población femenina e infantil empleada en el agro (Seefoó 2005). Los casos del tabaco y la floricultura son ejemplos al respecto.

En la producción tabacalera de Jujuy, si bien las fincas -dependiendo de su extensión- se sostienen con dos a cinco empleados permanentes, a medida que se avanza en el proceso productivo se hace necesario incorporar trabajadores transitorios, sobre todo en la época de cosecha, encañado y curado de la hoja de tabaco. Esto no quiere decir que la alta demanda de trabajo se traduzca en mejores salarios y condiciones laborales para atraer a los asalariados a las fincas, sino todo lo contrario. La búsqueda constante de mano de obra, la competencia y la alta rotación de los asalariados entre las fincas desdibujan el mercado de trabajo, haciendo más impersonales las relaciones, al punto de desconocer incluso para quién se está trabajando y a quién se emplea (Re 2007).

Al no existir mecanismos formales de tercerización, como lo son las empresas o cooperativas de trabajo ampliamente difundidas en otras regiones de Argentina9 ${ }^{9}$, el productor tabacalero jujeño pone en práctica diversas estrategias para asegurarse la fuerza de trabajo necesaria para la cosecha. Esta particularidad del mercado de trabajo hace que los productores salgan a buscar ellos mismos a los asalariados al pueblo. En otros casos, pueden mandar al capataz o al encargado, e incluso delegar esta tarea en contratistas. Estos últimos, si bien cumplen el típico rol de vincular la oferta con la demanda de empleo, no poseen mayor capital que un camión; en rigor, no corren con ningún tipo de riesgo ni de gasto, más que el combustible que demanda su vehículo (Re 2010).

Esta modalidad de contratación genera altos niveles de rotación y hace competir a los asalariados entre sí. Un cosechero de tabaco se ve sometido al cambio diario de empleadores, con lo cual pierde la idea de para quién trabaja y la posibilidad de realizar cualquier mecanismo formal de protesta. La mayoría de estos "jornaleros" que se mueven dentro de este circuito no cuentan con libreta de trabajo, ni cobertura social de ningún tipo. Los trabajadores que aquí se ofrecen, provienen de zonas de pobreza cercanas al área productora y de asentamientos periurbanos.

Como resultado de este proceso, prevalecen mecanismos informales de contratación y el uso de un sector intermediario con un rol diferenciado de organizador de la provisión de trabajadores. La prevalencia de estos mecanismos cuasi informales de vinculación también se manifiesta en las distintas contrataciones que un trabajador consigue a lo largo del ciclo productivo.

Para el caso de la floricultura comercial de exportación en México, el panorama y funcionamiento del mercado de trabajo local es similar al CAI tabacalero

9 Ejemplo de esta situación lo constituyen los contratistas de esquila en la provincia de Chubut y los contratistas intermediarios entre la agroindustria y los productores de yerba mate en Misiones (Aparicio y Re 2014). 
jujeño en Argentina. En la producción de flores de corte prevalecen una serie de contradicciones que se materializan en una situación de desigualdad entre grandes y pequeños productores, ya que la política estatal hacia el sector agrícola ha favorecido fundamentalmente la agricultura con mayor capitalización, es decir, a los productores con mayores recursos, mientras que ha desplazado las principales actividades económicas de las unidades familiares y campesinas, debido a que les es imposible competir en los mercados internacionales (Miranda 2012).

Por ello, los pequeños productores se han visto en la necesidad de buscar estrategias para diversificar sus opciones de ingresos encontrando nuevas fuentes de sobrevivencia en fenómenos como la migración y la incorporación al trabajo asalariado de más miembros de la familia en las actividades agroindustriales más dinámicas, tales como la floricultura comercial. El mercado de trabajo de la floricultura en el contexto local descrito constituye una importante condición estructural que brinda a nuevos actores, como mujeres, niños y niñas, facilidades para entrar y salir de este en forma sumamente flexible. La flexibilidad propia del mercado de trabajo local es una variable que condiciona distintas formas de participación laboral pero que al mismo tiempo deriva en condiciones precarias de empleo; la constante presencia de trabajadores temporales es un ejemplo de ello en este mercado de trabajo agrícola.

Como podemos ver, en ambos escenarios (producción de tabaco y flores) el mercado de trabajo se compone por una gran cantidad de asalariados y familias enteras dispuestas a emplearse en las temporadas de siembra/plantación, corte/ cosecha y poscosecha. En este proceso, se facilita e incluso se incentiva el trabajo infantil. Con diferentes modalidades, el trabajo de niñas y niños está presente en ambos procesos productivos, y representa una manifestación más de la constante precariedad de las condiciones laborales y bajos ingresos que imperan en la agricultura latinoamericana.

\section{El trabajo infantil en cada contexto en particular}

Una práctica social que no es nueva en el sector agrícola latinoamericano es el trabajo infantil, dado que tradicionalmente la estrategia de las familias rurales ha sido el trabajo de todos los miembros del hogar incluyendo a las niñas y los niños. La diferencia sustancial es que en la actualidad este grupo poblacional se emplean en contextos de creciente competitividad entre países y en actividades que requieren incorporar estrategias para posicionarse en los mercados internacionales.

$\mathrm{Al}$ respecto, como ya señalamos anteriormente, hay ciertos cultivos que por sus características productivas requieren de un uso intensivo de fuerza de trabajo estacional; tal es el caso de la producción intensiva de flores y de tabaco, vinculados a los mercados internacionales. En este sentido, estas ramas productivas se sitúan en los sectores menos regulados de la política agrícola común internacional, por lo que están sometidos de manera más fuerte a un capitalismo salvaje (Seefoó 2005).

Para el caso de la zona florícola del sur del Estado de México, los niños y las niñas jornaleras son el rostro oculto del mercado de trabajo local. No obstante, 
su participación en este mercado de trabajo es constante y se desarrolla en distintas modalidades.

La producción de flores de corte tiene importantes picos productivos que requieren demandar mano de obra de forma intensiva. Las fechas de mayor demanda de fuerza de trabajo se concentran en febrero, por la celebración del día de San Valentín, tanto en México como en Estados Unidos; igualmente, el mes de mayo es una importante época de reclutamiento laboral, pues en México se celebra el Día de la Madre; en octubre, que antecede a la tradicional celebración de Día de Muertos, se requiere también de abundante fuerza de trabajo. En los invernaderos y campos florícolas, es común observar en estas fechas la presencia de niños y niñas realizando tareas propias de dicha actividad productiva. Una de las causas inmediatas es la marcada participación femenina, la incorporación de las mujeres como cortadoras y embaladoras de flores se combina con las actividades domésticas de cuidado. Las mujeres llevan como acompañantes a hijos e hijas, ya que no cuentan con mecanismos que las sustituyan en el cuidado de los hijos mientras se incorporan al empleo. Esta es una práctica que marca el inicio de las actividades laborales de los niños y las niñas.

De hecho se han identificado modalidades de trabajo infantil invisibilizado detrás de la lógica de acompañamiento y ayuda a las mujeres cortadoras. No obstante, debido a los mecanismos de retribución que se acostumbran en las épocas de alta producción (pago a destajo o por unidad), el aporte de los niños y las niñas es sustancial para completar los ingresos de los trabajadores adultos. Un encargado o capataz entrevistado explica:

\footnotetext{
Los patrones son buenas personas, les dejan a las cortadoras que traigan a sus hijos, se arriesgan pero sí dejan que vengan los niños y ya es de ellas si quieren ayudar en el jornal o no... y uno como encargado no se mete en esas cosas, con eso se ayudan las cortadoras, acarrean más flor, y uno como encargado debe buscar que salga bien el trabajo y que salgan los pedidos, ése es el trabajo de uno, no es fácil que salga bien el trabajo (encargado de empresa, Villa Guerrero, México, 2010).
}

Asimismo, existe una naturalización generalizada al respecto. Tanto empleadores y capataces, como padres y madres de estos niños y niñas reconocen que sin la partición laboral de los menores de edad la productividad se vería merma$\mathrm{da}$, al igual que los ingresos de muchas familias.

Al ser una actividad productiva dirigida al consumo de un bien estético, prevalece un discurso generalizado sobre la necesidad de habilidades laborales tales como la delicadeza y la paciencia, adjudicadas tradicionalmente a lo femenino. Por ello, la contratación de mujeres, niñas y niños es una constante en las temporadas de alta producción. El gerente de la Asociación Mexicana de Floricultores, que tiene su sede en el municipio de Villa Guerrero, Estado de México, adjudica la contratación de niñas y niños a las características físicas de estos. Desde su perspectiva, los niños y las niñas al ser pequeños están más cerca del suelo por lo que se les facilitan algunas actividades como el corte de maleza y la limpieza del terreno (Miranda 2012).

En la floricultura de corte, el trabajo infantil es una práctica oculta pero de conocimiento generalizado. Por ejemplo, los intermediarios o contratistas 
incurren en prácticas que facilitan la entrada temprana de los niños y las niñas al trabajo agrícola. El siguiente testimonio de una cortadora da luces al respecto:

Ella tiene 11 pero como ya pasa por grande la pudieron contratar aparte, fue lo bueno porque le digo como ya pasa por grande el encargado quiso contratarla aparte. Y cómo le puedo decir, así ella lleva su propia flor y le pagan aparte... Ella va en sexto, pero estas semanas no va, ya sabe la maestra (cortadora en empresa floricultora, Villa Guerrero, México, 2010).

Esta modalidad de participación de los niños y las niñas en las empresas floricultoras es común. En las épocas de alta producción muchos de los niños y las niñas dejan de asistir a la escuela para ir a cortar o empacar flores. Las profesoras de las escuelas primarias y secundarias saben que la mayoría de los estudiantes faltan a clases en esas temporadas. Incluso hay fechas en que se suspenden las clases por falta de niños y niñas. Esto sucede en caso de que los días de San Valentín o el 10 de mayo o Día de la Madre caigan entre semana:

\begin{abstract}
Mira, aquí los padres no entienden, a la escuela llegan niños que trabajan en el corte y en el empaque, y faltan, desde los 7 u 8 años ya están yendo a trabajar. Te digo que los padres no entienden y nosotras ya sabemos, por eso no abarcamos los contenidos programáticos, no podemos avanzar en el conocimiento si no hay asistencia; y es complicado porque faltan en las semanas de corte y cuando vuelven están cansados, inquietos, es muy difícil... Nosotras ya sabemos: así es todos los años, y luego dicen que somos las maestras las que no trabajamos (directora escuela primaria, Villa Guerrero, México, 2010).
\end{abstract}

Este tipo de participación de los niños y las niñas en el mercado de trabajo de la floricultura muestra variaciones en la forma en que se experimenta el trabajo infantil y sus implicaciones. Cabe señalar que no significa lo mismo ser un niño o niña que trabaja de tiempo completo en una empresa, que un niño o niña que únicamente participa en la floricultura durante las temporadas de alta producción. O bien, los niños y niñas que únicamente ayudan en la unidad económica campesina expresan distintas vivencias que quienes trabajan para una empresa de tiempo completo.

\footnotetext{
Yo inicié contando los tallos cuando tenía 11, ahora ya corto y empaco; cada vez te ponen a más cosas, como vas aprendiendo; y como ya me pusieron en el corte ya mejor me quedé a trabajar para que descanse mi mamá de tantos gastos (cortadora de flor gerbera -15 años de edad-, Villa Guerrero, México, 2010).
}

Las complejidades de estos procesos se ven acentuadas aún más si se toma en consideración que esta práctica social cuenta con especificidades asociadas también con las condiciones de género. Las niñas tienen doble jornada en comparación con los niños, además de ejercer tareas propias de la floricultura como cortar, limpiar el terreno, barrer los residuos y empacar, al momento del almuerzo deben calentar y servir los alimentos para sus familiares y hermanos; de hecho, en los tiempos de descanso o en las horas muertas son los niños varones quienes aprovechan para jugar y distraerse en tanto que las niñas deben ejercer tareas de cuidado y servicio. 
Yo lo que cambiaría es que mi mamá ponga también a mi hermano a servir el almuerzo y a lavar los trastes, porque en veces cuando vamos al corte él bien que va a jugar y se echa sus cascaritas con los más grandes y yo no puedo, eso cambiaría porque él ni su relajo recoge (niña dedicada al desyerbe -10 años de edad-, Villa Guerrero, México, 2010).

En cuanto al cultivo de tabaco, es posiblemente una de las producciones de mayor requerimiento de mano de obra estacional y concentrada en determinados momentos del año (diciembre a marzo) cuando se produce la desfloración y la cosecha de las hojas. Ambas tareas manuales.

Al analizar detalladamente el mercado de trabajo, observamos que existen unos pocos trabajadores permanentes; estos son en su mayoría hombres que consiguen trabajos estables en las fincas tabacaleras. Ello responde a las necesidades del sector de tener, al menos, un trabajador permanente durante todo el año e ir contratando asalariados transitorios a medida que se avanza en el proceso de producción. Sin embargo, será durante los meses de la cosecha que se ponen en práctica toda una serie de estrategias tendientes a cubrir los requerimientos de mano de obra necesaria para cosechar, encañar y clasificar las hojas de tabaco (Re 2007). Es precisamente en este contexto donde el trabajo de mujeres, niños, niñas y adolescentes adquiere protagonismo.

En el área tabacalera de Perico del Carmen, Argentina, con base en una encuesta realizada en el $2007^{10}$ a 163 hogares de asalariados, se encontraron 124 personas menores de 19 años. Lo más significativo de los datos recogidos es que de ese universo, $10 \%$ correspondía a niños y niñas menores de 9 años que habían trabajado en algún momento del año. La participación de niños y niñas de entre 10 y 14 años en alguna actividad laboral representaba 30,3\% de su grupo etario, y entre los 15 y 19 años de edad, $63,2 \%$ ya había participado de alguna tarea productiva dentro del tabaco. Estos datos, sin lugar a duda, dan cuenta de un inicio precoz en el mundo laboral.

Se debe tener en cuenta, como ya mencionamos, que la actividad tabacalera va incrementando gradualmente la demanda de trabajadores hasta llegar a la época de desflore ${ }^{\text {II }}$ y cosecha, en los meses de verano y que coincide con la etapa de receso escolar. Este hecho obstaculiza el trabajo de las madres que no disponen de recursos económicos y sociales para conseguir donde dejar a los niños y niñas ${ }^{12}$.

Muchas trabajadoras han mencionado que cuando están con sus hijos es en el encañado/desencañado ${ }^{13}$ en la única tarea donde las emplean, ya que ellos

10 Realizada por el proyecto: "Tabaco, mercado de trabajo y cultura en Jujuy", bajo la dirección de Susana Aparicio y el financiamiento de la Universidad de Buenos Aires y la Superintendencia de Riesgos del Trabajo: "Programa de promoción de la investigación, formación y divulgación sobre riesgos del trabajo" (convocatoria 2005).

11 Desflore: comprende el corte de la flor de la planta y la aplicación manual de un agroquímico, acción que se realiza en cada una de las plantas y permite un mayor desarrollo de las hojas.

12 Si bien existen programas de "cuidado" promocionados por las principales instituciones del sector y el gobierno, muchos niños y niñas aún no han sido incorporados al sistema, incluso por decisiones personales.

13 El encañado es una de las tareas que se realiza luego de la cosecha; consiste en atar una a una las hojas de tabaco a una vara para ser llevadas luego a las estufas de secado. El desencañado es la acción inversa luego de completado el proceso. 
pueden colaborar en la labor. En cuanto a la contratación de mujeres, los empleadores sostienen que son ellas las más indicadas para determinados trabajos:

Las encañadoras por lo general son madres que no pueden ir al campo porque no tiene con quien dejar a sus hijos, tienen bebés o chicos chiquitos que las acompañan a los galpones. Las que desfloran son chicas más jóvenes y fuertes (productor tabacalero No 1, Perico del Carmen, Jujuy, Argentina, 2012).

Cuando hay trabajo se van las madres con los chicos a los galpones y las mesas de encañado, porque son muchas horas que no están en la casa y no pueden quedarse solos (productor tabacalero No 2, Perico del Carmen, Jujuy, Argentina, 2012).

La participación de niños y niñas en el trabajo también se puede observar dentro del propio hogar. Los hijos de los asalariados tabacaleros ayudan en las tareas domésticas con una tradicional división del trabajo por género: las hijas se encargan de cuidar a los más chicos, cocinar, lavar y limpiar la casa. La contratación de niños, niñas y adolescentes, muchas veces hijos de los mismos asalariados, es una práctica común dentro de las fincas tabacaleras. Tareas tales como encañar o desencañar las hojas de tabaco, a pesar de estar prohibido, son una práctica habitual en la actividad tabacalera aunque no sea reconocida:

No, no. Menores no se pueden hacer trabajar. Muchas veces sí, el padre o la madre le pide que ate, que desate (encañar hojas de tabaco para el secado). Hay fincas chicas en las que trabaja la familia. Que ayudan las familias (productor tabacalero № 3, Perico del Carmen, Jujuy, Argentina, 2012).

Otros testimonios dan cuenta del fenómeno, pero no se responsabilizan directamente de la contratación de niños y niñas:

El niño no tanto. Porque viste con el tema del trabajo infantil. Aparte los trabajos en las fincas son pesados. Chiquito por ahí, qué sé yo... yo cuando era chico me llevaban, pero como era hijo del dueño y a mí me gustaba, agarraba y me ponía a descargar. No me costaba nada. Pero no se utiliza chicos para nada prácticamente... Sí, bueno, de que sigue, sí. Pero chicos, chicos no. Niños no, absolutamente no. Si las esposas van con sus chiquitos y como no los pueden dejar con nadie van con sus chiquitos a jugar. Los que vienen con familia son los que vienen siempre de la finca de al lado. Como ellos figuran en blanco, los grandes dicen voy a buscar gente de ahí para que no me hagan problema. Entonces ellos sí se van con sus hijitos (productor tabacalero No 1, Perico del Carmen, Jujuy, Argentina, 2012).

En la situación particular de Jujuy (así como en las flores), donde se combina la existencia de un mercado de trabajo altamente demandante de mano de obra y las necesidades económicas propias de hogares con bajos ingresos y falta de trabajo durante todo el ciclo ocupacional, se genera el inicio temprano en la actividad de muchos menores. Sin embargo, la naturalización de estas labores y las prácticas de ayuda familiar invisibiliza sus tareas, incluso para las mismas familias de asalariados rurales:

Los chicos no trabajan; la única temporada que trabajan es la encañada cuando están de vacaciones (...) en el tiempo de cosecha me ayudan a encañar (asalariada de finca tabacalera -hija de capataz-, Perico del Carmen, Jujuy, Argentina, 2007). 
A pesar de ser una actividad donde existe un importante trabajo en pos de la erradicación del trabajo infantil y adolescente (es común ver carteles en los establecimientos que indican la prohibición del ingreso de menores) persiste la incorporación al trabajo, tanto por necesidades económicas de las familias como por su participación al acompañar a sus madres.

Por su parte, en la región florícola del sur del Estado de México, es escasa la participación del sector estatal en materia de políticas públicas que beneficien a la infancia. El sistema municipal de desarrollo integral de la familia realiza nulas actividades frente a la presencia del trabajo infantil en el agro, argumentando que los únicos programas que llevan a cabo son relativos a la niñez en situación de calle, que no aplica a una realidad rural donde no existen niños y niñas en dicha condición.

\section{Reflexiones finales}

A la luz de la descripción realizada de la producción de tabaco en Argentina y de flores en México, se retoma la idea apuntada inicialmente sobre las transformaciones productivas que han experimentado ambos sectores para adecuarse a los requerimientos de competitividad globales, lo que ha derivado en la forma de gestionar la mano de obra, incluyendo la participación infantil.

Como podemos ver, ambas producciones son atravesadas por un mismo proceso: la incorporación de un nuevo patrón tecnológico de producción impuesto por los CAI, que impacta de manera generalizada sobre la estructura social de cada actividad.

Cada una de las modalidades analizadas presenta la inserción en la agricultura de niños y niñas. Esta implica distintas condiciones en que se realiza pero un mismo denominador, un gran mercado de trabajo -compuesto mayormente por familias enteras- dispuesto a emplearse en las temporadas de mayor demanda.

Así, el análisis y comparación de ambos mercados de trabajo permiten establecer que detrás del trabajo infantil subyace el interés de los empleadores por posicionarse en los mercados globales, procesos que requieren de una mano de obra flexible, temporal, barata y disponible.

Un aspecto que comparten los dos casos descritos es el relacionado con la inexistencia de mecanismos de soporte para las tareas de cuidado de los miembros más pequeños de las familias. Esta situación deriva en la necesidad de acompañamiento de los niños y las niñas a los espacios laborales de las madres u otros trabajadores adultos. Así, en estos contextos agroindustriales, el acompañamiento representa en muchos casos el primer acercamiento de los niños y las niñas a las actividades productivas agrícolas.

Otro importante elemento que comparten los complejos agroindustriales descritos es la pobreza y precariedad material a la que se enfrentan las familias trabajadoras de los ámbitos rurales, lo que se entrelaza con las modalidades de pago a destajo y por unidad que se acostumbran en los modelos agroindustriales y que devienen en la necesidad de aumentar el número de miembros de las familia para completar los ingresos totales. Por lo que en la producción de tabaco en Argentina y en la floricultura en México, la participación de los 
niños y las niñas es una práctica que responde en cierta medida a la lógica de supervivencia de la población rural.

Por otro lado, tanto en la producción de tabaco como en la producción de flores, la aportación que hacen las niñas y los niños además de ser invisibilizada es infravalorada bajo el argumento de la ayuda que comprende tareas marginales de menor envergadura en comparación con el trabajo de los adultos.

La infravaloración de la aportación económica que hacen estos niños y niñas a la producción florícola y tabacalera es importante en cuanto realizan las actividades cuyo costo no es pagado en su totalidad por los empleadores. Barrer, limpiar, desyerbar, cargar, encañar son actividades vistas como fáciles y marginales que al ser efectuadas por las niñas y los niños funcionan como un importante minimizador de costos para los empleadores y en muchos casos una importante entrada de ingresos a la economía familiar.

El modelo de gestión flexible de la fuerza de trabajo que prevalece tanto en la actividad tabacalera y como en la florícola, y que incluye la presencia de niños y niñas como trabajadores representa una oportunidad para garantizar el volumen de mano de obra a bajo costo necesaria en los picos productivos. La temporalidad, la flexibilidad y la disponibilidad que ofrecen los niños y las niñas es un elemento clave que garantiza la viabilidad de estos cultivos y su capacidad para hacer frente a la competitividad de los mercados agrícolas globalizados.

Una reflexión obligada en cuanto al trabajo infantil en el tabaco y en las flores es el papel del Estado como garante de los derechos de los niños y las niñas en ambos contextos locales. Para el caso de la floricultura mexicana, el Estado omite su responsabilidad, pues no garantiza que se cumpla la legislación establecida en materia de trabajo infantil y además refuerza esta práctica al promover su política de ayuda crediticia a todos aquellos productores que comprueben competitividad a escala internacional y posibilidades de generación de divisas, lo que constituye un mecanismo que profundiza la utilización de esquemas laborales flexibles y feminizados. Para el caso de la producción tabacalera, aun cuando es una zona en la que se realizan acciones tendientes a erradicar el trabajo infantil, los empleadores y las familias han podido encontrar mecanismos que habilitan la presencia y aporte productivo de los niños y las niñas.

Finalmente, resulta de suma importancia hacer visible tanto el aporte económico de las tareas que realizan los niños y las niñas en ambos complejos agroindustriales, como la contribución del trabajo infantil en los sectores agrícolas, para con ello orientar políticas públicas que consideren al trabajo infantil como uno de los principales procesos de reproducción de desigualdades en América Latina.

\section{Referencias bibliográficas}

Aparicio, Susana. El trabajo infantil en la Argentina. Análisis y desafíos para la política pública. Buenos Aires: $1^{\mathrm{a}}$ edición, Oficina de la OIT en Argentina; Ministerio de Trabajo, Empleo y Seguridad Social, 2007. 
Aparicio, Susana, y Daniel Re. "Cambios en el mercado de trabajo tabacalero y formas de contratación de asalariados transitorios». En Nuevas formas de contratación en el trabajo agrario, compilado por Susana Aparicio y Roberto Benencia, 58-71. Buenos Aires: Ciccus, 2014.

Bartra, Roger. Estructura agraria y clases sociales en México D.F. México D.F.: Ediciones Era, 1974.

Corradini, Eugenio. Caracterización del sector productor tabacalero en la República Argentina. Buenos Aires: Facultad de Ciencias Agrarias, UCA, 2005.

Fenner, Justus y Thomas Gebauer. Las flores de la muerte. Ensayo sobre la floricultura mexicana. México D.F.: Ediciones GEA, 1992.

Instituto Nacional de Tecnología Agropecuaria (INTA). «Evaluación económica de sistemas de producción de tabaco Virginia para dos tipos de curado: convencional y bulk-curing, en los valles templados de Jujuy». En Economía de los cultivos industriales: algodón, caña de azúcar, maní, tabaco, té y yerba mate, de Rodolfo Bongiovanni. Buenos Aires: Instituto Nacional de Tecnología Agropecuaria, 2008.

Miranda, Sarai. Trabajo infantil y floricultura. Imbricaciones entre las necesidades de reproducción del capital y la supervivencia de las unidades domésticas. El caso de Villa Guerrero, Estado de México. Tesis doctoral en Estudios de Población. México D.F.: El Colegio de México, 2012.

Morett, Jesús y Celsa Cosío. Los jornaleros agrícolas en México, D.F. México D.F.: Universidad Autónoma Chapingo-Diana, 2004.

Murmis, Miguel. «Incluidos y excluidos en la reestructuración del agro latinoamericano». Debate Agrario. Análisis y Alternativas, nº 18 (abril 1994): 101-132.

Organización Internacional del Trabajo (OIT). «Erradicar el trabajo infantil agrícola: una forma de romper el ciclo de la pobreza». OIT, 2013. http://www.ilo. org/santiago/sala-de-prensa/WCMS_229079/lang--es/index.htm (último acceso: 18 de febrero de 2015).

Orozco, María. «Competitividad local de la agricultura ornamental en México». Ciencia Ergo Sum, Vol. 10, n 1 (marzo-junio 2007): 128-142.

Re, Daniel. «La producción tabacalera en Jujuy. Mercado de trabajo y productores medios". Ponencia presentada en el V Congreso Latinoamericano de Sociología del Trabajo: hacía una nueva civilización del trabajo, de la Asociación Latinoamericana de Sociología del Trabajo (Alast). Montevideo: 10 de abril 2007.

Re, Daniel. La producción de tabaco Virginia en la provincia de Jujuy y su impacto sobre la estructura social agraria. ¿Movilidad social de productores? Tesis doctoral en Ciencias Sociales. Buenos Aires: Facultad De Ciencias Sociales, UBA, 2013.

Rossi, Carlos y Carlos León. «Temas fundamentales para la inserción de los pequeños productores agropecuarios en cadenas comerciales». Revista Interdisciplinaria de Estudios Agrarios, $\mathrm{n}^{\circ} 28$ (1er semestre 2008): 3-57.

Seefoó, José. La calidad es nuestra, la intoxicación... ¡de usted! Atribución de la responsabilidad en las intoxicaciones por plaguicidas agrícolas Zamora, Michoacán, 1997-2000. Morelia: El Colegio de Michoacán, 2005.

Vargas, Jorge. El desarrollo local en el contexto de la globalización. Toluca: INAPUAEM, 2007. 


\section{Entrevistas}

Encargado de supervisar el trabajo de corte de las trabajadoras en el área de invernadero. 34 años. Entrevista realizada por Sarai Miranda Juárez, el 25 de enero de 2010, Villa Guerrero, Estado de México.

Cortadora de rosas en empresa floricultora. 28 años. Entrevista realizada por Sarai Miranda Juárez, 25 de enero de 2010, Villa Guerrero, Estado de México.

Directora de escuela primaria. 43 años. Entrevista realizada por Sarai Miranda Juárez, 18 de abril de 2010, Villa Guerrero, Estado de México.

Cortadora de flores. 15 años. Entrevista realizada por Sarai Miranda Juárez, 10 de febrero de 2010, Villa Guerrero, Estado de México.

Niña dedicada el desyerbe de los invernaderos. Cuarto año de primaria. 10 años de edad. Entrevista realizada por Sarai Miranda Juárez, 12 de febrero de 2010, Villa Guerrero, Estado de México.

Productor tabacalero $\mathrm{N}^{\circ} 1.49$ años. Entrevista realizada por Daniel Re, 10 de diciembre de 2012, Perico del Carmen, Jujuy, Argentina.

Productor tabacalero $\mathrm{N}^{\circ} 2.58$ años. Entrevista realizada por Daniel Re, 18 de julio de 2012, El Carmen Monterrico, Jujuy, Argentina.

Productor tabacalero $\mathrm{N}^{\circ} 3.61$ años. Entrevista realizada por Daniel Re, 30 de julio de 2012, El Carmen Monterrico, Jujuy, Argentina.

Asalariada de finca tabacalera, hija de capataz. 20 años. Entrevista realizada por Daniel Re, 15 de diciembre de 2007, Perico del Carmen, Jujuy, Argentina. 